\title{
Lattice Boltzmann simulations for flow and heat/mass transfer problems in a three-dimensional porous structure
}

\author{
M. Yoshino ${ }^{1, *}$ and T. Inamuro ${ }^{2}$ \\ ${ }^{1}$ Department of Mechanical Systems Engineering, Shinshu University, Nagano 380-8553, Japan \\ ${ }^{2}$ Department of Chemical Engineering, Kyoto University, Kyoto 606-8501, Japan
}

\section{SUMMARY}

The lattice Boltzmann method (LBM) for a binary miscible fluid mixture is applied to problems of transport phenomena in a three-dimensional porous structure. Boundary conditions for the particle distribution function of a diffusing component are described in detail. Flow characteristics and concentration profiles of diffusing species at a pore scale in the structure are obtained at various Reynolds numbers. At high Reynolds numbers, the concentration profiles are highly affected by the flow convection and become completely different from those at low Reynolds numbers. The Sherwood numbers are calculated and compared in good agreement with available experimental data. The results indicate that the present method is useful for the investigation of transport phenomena in porous structures. Copyright (c) 2000 John Wiley \& Sons, Ltd.

KEY WORDS: lattice Boltzmann method (LBM); porous structure; heat/mass transfer

\footnotetext{
*Correspondence to: Department of Mechanical Systems Engineering, Faculty of Engineering, Shinshu University, 4-17-1 Wakasato, Nagano 380-8553, Japan.

Tel.: +81-26-269-5116; Fax: +81-26-269-5109; E-mail address: masato@gipwc.shinshu-u.ac.jp
}

Received 7 January 2002 


\section{INTRODUCTION}

Transport phenomena in porous media are important subjects of many science and engineering fields. The problems of flow and heat/mass transfer in porous media are found in hydraulics, biomechanics, soil mechanics, geothermal operations, packed-bed chemical reactors, drying processes, and so on. In the past studies, volume-averaged approaches are usually used to obtain macroscopic properties such as pressure drops, effective thermal conductivities and effective mass diffusivities in porous media. For fluid flows through porous media, for example, Ergun [1] proposed the empirical equation based on experimental data to estimate pressure drops through packed columns. As for heat transfer in porous media, on the other hand, Vafai and Tien [2] utilized the local volume-averaging technique and investigated the boundary and inertial effects upon flow and heat transfer in porous media. Buonanno and Carotenuto [3] proposed a method to calculate the effective thermal conductivity of a two-phase isotropic porous medium by means of a volume averaging technique.

However, for complex porous structures with spatially non-uniform porosity, it is essential to investigate microscopic behaviors occurring at a pore scale in the porous structure. Particularly, as for heat/mass transfer in porous media at high Reynolds numbers, the volume-averaged approaches often give incorrect estimates of macroscopic properties due to the appearance of unsteady vortices. In addition, according to [4-7], one should take account of the effect of dispersion, which is a convection-diffusion phenomenon peculiar to fluid flows through porous media at high Péclet numbers. Thus, it is needed to investigate the relation between heat/mass transfer and fluid flow characteristics from the microscopic point of view.

In recent years, the lattice Boltzmann method (LBM) [8-11] has been used for many kinds of simulations of incompressible viscous flows. The main advantages of the LBM over 
conventional Navier-Stokes codes are the simplicity of the algorithm and the flexibility for complex boundaries $[12,13]$. Hence it is particularly successful in fluid applications involving dynamic interface and complex boundaries such as porous media. As for fluid flows through porous media, for example, Cancelliere et al. [14] studied the permeability as a function of solid fraction in a system of randomly positioned spheres by using the LBM. Inamuro et al. $[15,16]$ carried out LB simulations of fluid flows in a three-dimensional porous structure relatively at high Reynolds numbers, and investigated unsteady flow characteristics at a pore scale in the structure. Zeiser et al. [17] employed the LBM and examined the pressure drops in fixed-bed reactors, taking account of all effects of flow characteristics caused by the radial and circumferential inhomogeneities of the packings.

On the other hand, the LBM has also been applied to problems of viscous flows with heat and mass transfer [18-22]. Recently, Inamuro et al. [23] have proposed an LBM for a binary miscible fluid mixture with a simpler equilibrium distribution function for the concentration than that of $[21,22]$, and have demonstrated the validity and the accuracy of the method theoretically and numerically. This LBM can also be applied to thermal fluid systems by utilizing the formal analogy between the concentration of a diffusing component and the temperature of the fluid. Therefore, it is considered that the proposed LBM is useful and efficient for the microscopic investigation of transport phenomena in porous structures.

In this paper, the above-mentioned LBM for a binary miscible fluid mixture is applied to the problems of transport phenomena in a three-dimensional porous structure in order to investigate the characteristics of heat/mass transfer at a pore scale in the structure. Also, boundary conditions of the particle distribution function of a diffusing component are presented in detail. 


\section{LATTICE BOLTZMANN METHOD}

In the LBM, a modeled gas, which is composed of identical particles whose velocities are restricted to a finite set of vectors, is considered and the evolution of the particle population at each lattice site in physical spaces is computed. The main advantage of the LBM over other numerical approaches is considered as follows.

In the case of conventional numerical methods such as the finite difference method, the discretized macroscopic equations are directly solved. Since the macroscopic equations are nonlinear and the solutions of nonlinear equations are highly related to boundaries, it should be difficult to solve the equations especially if the physical system has complex geometries. Moreover, when the incompressible Navier-Stokes set is computed by using the conventional methods, the pressure satisfies a Poisson equation and solving this equation for the pressure often produces numerical difficulties requiring special treatment such as iteration or relaxation (e.g., see [24]). In the LBM, on the other hand, one takes advantage of an analogy with the kinetic theory of gases and solves the kinetic equation which is called the lattice Boltzmann equation. Due to the simple linear form of the differential term of kinetic equation, the algorithm of the LBM is very simple. Therefore, it is considered that the LBM can be an efficient and attractive tool for simulating the fluid flows including complex phenomena.

\subsection{Method of Computation}

The three-dimensional fifteen-velocity model [11] is used in the following calculations, where the physical space is divided into cubic lattices. Hereafter, non-dimensional variables, which are defined by a characteristic length $L$, a characteristic particle speed $c$, a characteristic time scale $t_{0}=L / U$ where $U$ is a characteristic flow speed, a reference density $\rho_{0}$ and a reference 
mass concentration $\rho_{\sigma 0}$ are used as in $[23,25]$. The fifteen-velocity model has the following velocity vectors:

$$
\begin{aligned}
& {\left[\mathbf{c}_{1}, \mathbf{c}_{2}, \mathbf{c}_{3}, \mathbf{c}_{4}, \mathbf{c}_{5}, \mathbf{c}_{6}, \mathbf{c}_{7}, \mathbf{c}_{8}, \mathbf{c}_{9}, \mathbf{c}_{10}, \mathbf{c}_{11}, \mathbf{c}_{12}, \mathbf{c}_{13}, \mathbf{c}_{14}, \mathbf{c}_{15}\right]} \\
& =\left[\begin{array}{rrrrrrrrrrrrrrrrr}
0 & 1 & 0 & 0 & -1 & 0 & 0 & 1 & -1 & 1 & 1 & -1 & 1 & -1 & -1 \\
0 & 0 & 1 & 0 & 0 & -1 & 0 & 1 & 1 & -1 & 1 & -1 & -1 & 1 & -1 \\
0 & 0 & 0 & 1 & 0 & 0 & -1 & 1 & 1 & 1 & -1 & -1 & -1 & -1 & 1
\end{array}\right] .
\end{aligned}
$$

In the following, we consider a mass transfer problem in a binary miscible fluid mixture under the condition that the fraction of diffusing component, $\sigma$-species, is negligibly small. However, it should be noted that the following analysis can be applied to heat transfer problems by taking advantage of the formal analogy between the mass concentration of the $\sigma$-species and the temperature of the fluid. The evolution of the particle distribution functions, $f_{i}(\mathbf{x}, t)$ for the fluid and $g_{i}(\mathbf{x}, t)$ for the $\sigma$-species, with velocity $\mathbf{c}_{i}$ at the point $\mathbf{x}$ and time $t$ is computed by the following equations [23]:

$$
\begin{aligned}
f_{i}\left(\mathbf{x}+\mathbf{c}_{i} \Delta x, t+\Delta t\right)-f_{i}(\mathbf{x}, t) & =-\frac{1}{\tau_{f}}\left[f_{i}(\mathbf{x}, t)-f_{i}^{\mathrm{eq}}(\mathbf{x}, t)\right] \\
g_{i}\left(\mathbf{x}+\mathbf{c}_{i} \Delta x, t+\Delta t\right)-g_{i}(\mathbf{x}, t) & =-\frac{1}{\tau_{g}}\left[g_{i}(\mathbf{x}, t)-g_{i}^{\mathrm{eq}}(\mathbf{x}, t)\right]
\end{aligned}
$$

for $i=1,2,3, \ldots, 15$, where $\Delta x$ is a spacing of the cubic lattice, $\Delta t$ is a time step, $f_{i}^{\text {eq }}$ and $g_{i}^{\text {eq }}$ are equilibrium distribution functions, and $\tau_{f}$ and $\tau_{g}$ are single relaxation times which are of $O(1)$. Note that $\Delta t$ is chosen so that the particles travel one lattice spacing during the time step. Suitable equilibrium distribution functions of the fluid mixture are given by $[11,23]$

$$
\begin{aligned}
f_{i}^{\mathrm{eq}} & =E_{i} \rho\left[1+3 \mathbf{c}_{i} \cdot \mathbf{u}+\frac{9}{2}\left(\mathbf{c}_{i} \cdot \mathbf{u}\right)^{2}-\frac{3}{2} \mathbf{u} \cdot \mathbf{u}\right], \\
g_{i}^{\mathrm{eq}} & =E_{i} \rho_{\sigma}\left(1+3 \mathbf{c}_{i} \cdot \mathbf{u}\right),
\end{aligned}
$$

Copyright (C) 2000 John Wiley \& Sons, Ltd.

Int. J. Numer. Meth. Fluids 2000; 00:1-6 
for $i=1,2,3, \ldots, 15$, where $E_{1}=2 / 9, E_{2}=E_{3}=\cdots=E_{7}=1 / 9$ and $E_{8}=E_{9}=\cdots=$ $E_{15}=1 / 72$. The density $\rho$ of the fluid, the flow velocity $\mathbf{u}$ and the mass concentration $\rho_{\sigma}$ of the $\sigma$-species are defined in terms of the particle distribution functions as follows:

$$
\begin{aligned}
\rho & =\sum_{i=1}^{15} f_{i}, \\
\mathbf{u} & =\frac{1}{\rho} \sum_{i=1}^{15} f_{i} \mathbf{c}_{i}, \\
\rho_{\sigma} & =\sum_{i=1}^{15} g_{i},
\end{aligned}
$$

and the pressure $p$ is related to the density $\rho$ by

$$
p=\frac{1}{3} \rho .
$$

\subsection{Governing Equations for Macroscopic Variables}

Hereafter, the summation convention is used for the subscript $\gamma$ and $\delta(\gamma, \delta=x, y, z)$. As shown in [23, 25], applying asymptotic theory [26] to Equations (2), (3) and (6)-(9) with (4) and (5), we find that

$$
\mathbf{u}^{\prime}=\varepsilon \mathbf{u}^{(1)}+\varepsilon^{2} \mathbf{u}^{(2)}, \quad p^{\prime}=\varepsilon^{2} p^{(2)}+\varepsilon^{3} p^{(3)}, \quad \rho_{\sigma}^{\prime}=\rho_{\sigma}^{(0)}+\varepsilon \rho_{\sigma}^{(1)},
$$

with $\varepsilon$ being a small parameter which is of the same order as $\Delta x$, satisfy

$$
\begin{aligned}
\frac{\partial u_{\gamma}^{\prime}}{\partial x_{\gamma}} & =0 \\
S t \frac{\partial u_{\gamma}^{\prime}}{\partial t}+u_{\delta}^{\prime} \frac{\partial u_{\gamma}^{\prime}}{\partial x_{\delta}} & =-\frac{\partial p^{\prime}}{\partial x_{\gamma}}+\nu \frac{\partial^{2} u_{\gamma}^{\prime}}{\partial x_{\delta}^{2}} \\
S t \frac{\partial \rho_{\sigma}^{\prime}}{\partial t}+u_{\gamma}^{\prime} \frac{\partial \rho_{\sigma}^{\prime}}{\partial x_{\gamma}} & =D_{\sigma} \frac{\partial^{2} \rho_{\sigma}^{\prime}}{\partial x_{\gamma}^{2}}
\end{aligned}
$$

Copyright (C) 2000 John Wiley \& Sons, Ltd.

Int. J. Numer. Meth. Fluids 2000; 00:1-6 
where $S t(=U / c)$ is the Strouhal number, and the kinematic viscosity $\nu$ of the fluid and the mass diffusivity $D_{\sigma}$ of the $\sigma$-species in the fluid are given by

$$
\begin{aligned}
\nu & =\frac{1}{3}\left(\tau_{f}-\frac{1}{2}\right) \Delta x, \\
D_{\sigma} & =\frac{1}{3}\left(\tau_{g}-\frac{1}{2}\right) \Delta x .
\end{aligned}
$$

Equations (10)-(12) correspond to the continuity equation, the Navier-Stokes equations for the incompressible fluid and the convection-diffusion equation for the concentration of the $\sigma$-species, respectively. Therefore, it is found that using Equations (2)-(9) one can obtain the flow velocities, the pressure gradient for incompressible fluid and the mass concentration of diffusing species with relative errors of $O\left(\varepsilon^{2}\right)$. Also, it can be shown that the mass flux of $\sigma$-species, $\mathbf{j}_{\sigma}^{\prime}=\varepsilon \mathbf{j}_{\sigma}^{(1)}+\varepsilon^{2} \mathbf{j}_{\sigma}^{(2)}$, is given by [27]

$$
\mathbf{j}_{\sigma}^{\prime}=\rho_{\sigma}^{\prime}\left(\mathbf{u}_{\sigma}^{\prime}-\mathbf{u}^{\prime}\right)
$$

where $\mathbf{u}_{\sigma}^{\prime}=\varepsilon \mathbf{u}_{\sigma}^{(1)}+\varepsilon^{2} \mathbf{u}_{\sigma}^{(2)}$ is defined as follows:

$$
\mathbf{u}_{\sigma}^{\prime}=\frac{1}{\rho_{\sigma}^{\prime}} \sum_{i=1}^{15} g_{i} \mathbf{c}_{i} .
$$

In the case of heat transfer problems, the temperature $T$, the thermal diffusivity $\alpha$ and heat flux $\mathbf{q}$ of the fluid are given by Equations (8), (14) and (15) with (16), respectively. Moreover, the thermal conductivity $k$ is given by [27]

$$
k=\frac{1}{3} \tau_{g} \Delta x .
$$

\subsection{Comparison between $L B M$ and other numerical methods}

To compare the accuracy and computing time of the LBM with those of other numerical methods, we calculate natural convection flows in a square cavity with insulated top and bottom walls and with side walls maintained at constant but different temperatures, as shown 
in Figure 1. It is assumed that the temperature of the left-hand side wall is higher than that of the right-hand side wall. The Boussinesq approximation is used for the gravitational term. In the calculations, the two-dimensional nine-velocity model $[11,28]$ is employed for simplicity, but the basic theory and equations are the same as those for the fifteen-velocity model except values of the coefficients, $E_{i}$.

Figure 2 shows results for a case with $R a=10^{5}$ and $\operatorname{Pr}=0.71$ calculated on an $80 \times 80$ lattice, where $R a$ and $\operatorname{Pr}$ are Rayleigh and Prandtl numbers, respectively. It is seen that two clockwise rotational regions appear in the cavity and the temperature field is distorted by the flow. The accuracy of the result is checked by examining the mean Nusselt number $N u$, which is defined as the ratio of the actual heat flux across the cavity to the heat flux by pure conduction without flows. The present results give $N u=4.50$, which agrees within $0.5 \%$ with the grid-independent value of $N u=4.52164$ found by Hortmann et al. [29] using the finite volume multigrid method. In addition, the velocity vectors and the isotherms are in good agreement with their results. As for the computing time, the calculation requires 50 minutes to obtain the steady-state results on a Pentium 4 processor with $1.4 \mathrm{GHz}$ clock speed. Next, as an example for comparison, the same problem is calculated by a fourth-order finite difference method (FDM) based on the SIMPLER algorithm [30]. The calculated mean Nusselt number is $N u=4.56$ and slightly inferior to the result by the LBM. Also, the calculation by the FDM requires 69 minutes on the above-mentioned computer, and hence it takes about 1.4 times as much as the LBM to obtain almost the same accurate results. It should be noted that in the case of problems with complicated geometries such as porous structures, these differences will be more significant than in such a cavity flow problem. Thus, the LBM is more accurate and efficient than the conventional numerical methods in complex flow problems.

Copyright @ 2000 John Wiley \& Sons, Ltd.

Int. J. Numer. Meth. Fluids 2000; 00:1-6

Prepared using fldauth.cls 


\section{PROBLEM}

We consider the problem of flow and mass transfer in a three-dimensional porous structure shown in Figure 3. There exist nine identical spherical bodies in a rectangular domain whose size is $L_{y}=L_{z}=0.945 L_{x}$. The body is made up of a lattice block. The equivalent diameter $d_{\mathrm{p}}$ of the bodies is $0.403 L_{x}$, which is determined by the same method as in [15]. Then the porosity of the structure is 0.654 . The centers of the bodies are located at $\left(x / L_{x}, y / L_{y}, z / L_{z}\right)=$ $(0.21,0.29,0.22),(0.21,0.74,0.81),(0.22,0.71,0.22),(0.23,0.32,0.80), \quad(0.48,0.49,0.49)$, $(0.75,0.80,0.29),(0.78,0.23,0.70),(0.78,0.78,0.70)$ and $(0.80,0.23,0.29)$.

A periodic boundary condition with pressure difference is used at the inlet and outlet. A slip wall condition is applied to the other sides of the domain.

\section{BOUNDARY CONDITIONS}

Boundary conditions in terms of the distribution function for $\sigma$-species are described. Boundary conditions for the fluid are referred to [15].

\subsection{On the Body}

On the body, two types of boundary condition for $\sigma$-species are considered. One is the case that the concentration of $\sigma$-species is given at the surface of the body, and the other is the case that the normal mass flux of $\sigma$-species is zero at the surface. At a boundary node on the body, let $\mathbf{n}$ be the unit normal vector along the line connecting the node with the center of the body. The distribution functions of $\sigma$-species such that $\mathbf{c}_{i} \cdot \mathbf{n}>0$ are unknown at the boundary node. When the concentration of $\sigma$-species is given at the boundary node, the unknown distribution 
functions are assumed to be given by

$$
g_{i}=E_{i} \rho_{\sigma}^{\prime} \quad \text { for } \quad \mathbf{c}_{i} \cdot \mathbf{n}>0
$$

where $\rho_{\sigma}^{\prime}$ is an unknown parameter. Note that the counter slip velocity [31] is set to zero so as to maintain numerical stability at high Reynolds numbers [15]. The unknown parameter is determined so that the concentration of $\sigma$-species at the boundary node is equal to a given value $\left.\rho_{\sigma}\right|_{\mathrm{w}}$. Substituting Equation (18) and the known distribution functions $g_{i}$ for $\mathbf{c}_{i} \cdot \mathbf{n} \leq 0$ into Equation (8), the unknown parameter $\rho_{\sigma}^{\prime}$ is specified as follows:

$$
\rho_{\sigma}^{\prime}=\frac{\left.\rho_{\sigma}\right|_{\mathrm{w}}-\sum_{i\left(\mathbf{c}_{i} \cdot \mathbf{n} \leq 0\right)} g_{i}}{\sum_{i\left(\mathbf{c}_{i} \cdot \mathbf{n}>0\right)} E_{i}} .
$$

On the other hand, when the normal mass flux of $\sigma$-species is zero at the boundary node, the unknown distribution functions are also assumed to be Equation (18). From Equations (15) with (16), the normal mass flux $j_{\sigma n}\left(=\mathbf{j}_{\sigma} \cdot \mathbf{n}\right)$ on the body at rest is given by

$$
j_{\sigma n}=\sum_{i=1}^{15} g_{i} \mathbf{c}_{i} \cdot \mathbf{n}
$$

Hence, substituting Equation (18) and the known distribution functions $g_{i}$ for $\mathbf{c}_{i} \cdot \mathbf{n} \leq 0$ into Equation (20), the unknown parameter $\rho_{\sigma}^{\prime}$ is specified as follows:

$$
\rho_{\sigma}^{\prime}=-\frac{\sum_{i\left(\mathbf{c}_{i} \cdot \mathbf{n} \leq 0\right)} g_{i} \mathbf{c}_{i} \cdot \mathbf{n}}{\sum_{i\left(\mathbf{c}_{i} \cdot \mathbf{n}>0\right)} E_{i} \mathbf{c}_{i} \cdot \mathbf{n}} .
$$

\subsection{On the Side of Domain}

On the sides of the domain except for the inlet and outlet, we assume that the normal mass flux of $\sigma$-species is equal to zero. Thus, the above-mentioned boundary condition on the body with zero mass flux is also applied to this case. For example, at the lattice node on the face 
CDHG in Figure 3, we express the unknown distribution functions $g_{3}, g_{8}, g_{9}, g_{11}$ and $g_{14}$ by using Equation (18). The unknown parameter $\rho_{\sigma}^{\prime}$ is given by Equation (21), i.e.,

$$
\rho_{\sigma}^{\prime}=6\left(g_{6}+g_{10}+g_{12}+g_{13}+g_{15}\right)
$$

On the corner line, e.g., on the line $\mathrm{CG}, g_{3}, g_{4}, g_{8}, g_{9}, g_{10}, g_{11}, g_{14}$ and $g_{15}$ are unknown distribution functions. Since $g_{10}, g_{11}, g_{14}$ and $g_{15}$ are the distribution functions whose velocity points from the outer to outer region, one can not determine these unknown distribution functions even though the line is regarded as a common part of the two faces. In the following calculations, all the distribution functions including known distribution functions on the CG are set to the averaged value of the corresponding distribution functions at the two nearest neighboring lattice nodes in the $y$ - and $z$-directions. The same procedure is used on the other three corner lines and at every vertex from $\mathrm{C}$ through J.

\subsection{At the Inlet and Outlet}

At the inlet and outlet, a periodic boundary condition with a concentration difference is assumed. Hereafter, the subscript 'in' and 'out' represent quantities at the inlet and outlet, respectively. It is noted that under the periodic boundary condition, the concentration distribution of $\sigma$-species at the inlet is unknown quantity in advance. In the following calculations, the concentration difference $\Delta \rho_{\sigma}$ between the inlet and outlet is specified.

The unknown distribution functions at the inlet and outlet are determined as follows. At the inlet, the unknown distribution functions are $g_{2}, g_{8}, g_{10}, g_{11}$ and $g_{13}$. Taking account of the form of the equilibrium distribution functions given by Equation (5), we assume that the unknown distribution functions at the inlet can be written by adding constant values to the 
corresponding known distribution functions at the outlet as follows:

$$
\left.g_{i}\right|_{\text {in }}=\left.g_{i}\right|_{\text {out }}+E_{i}\left(K_{1}+c_{i x} K_{2}+c_{i y} K_{3}+c_{i z} K_{4}\right) \quad \text { for } i=2,8,10,11,13,
$$

where $K_{1}-K_{4}$ are constants, and $c_{i x}, c_{i y}$ and $c_{i z}$ are the $x-, y$ - and $z$-components of the velocity vector $\mathbf{c}_{i}$, respectively. Similarly, at the outlet, the unknown distribution functions $g_{5}, g_{9}, g_{12}, g_{14}$ and $g_{15}$ are assumed to be written by subtracting constant values from the corresponding known distribution functions at the inlet:

$$
\left.g_{i}\right|_{\text {out }}=\left.g_{i}\right|_{\text {in }}-E_{i}\left(K_{1}+c_{i x} K_{2}+c_{i y} K_{3}+c_{i z} K_{4}\right) \quad \text { for } i=5,9,12,14,15 .
$$

Then the constant values $K_{1}-K_{4}$ are determined by the following conditions. First, the concentration difference of $\sigma$-species between the inlet and outlet is equal to the given $\Delta \rho_{\sigma}\left(=\left.\rho_{\sigma}\right|_{\text {out }}-\left.\rho_{\sigma}\right|_{\text {in }}\right)$. By using Equation (8), we obtain

$$
\sum_{i=1}^{15}\left(\left.g_{i}\right|_{\text {out }}-\left.g_{i}\right|_{\text {in }}\right)=\Delta \rho_{\sigma}
$$

Next, considering the mass flux of $\sigma$-species at the inlet and outlet and using Equations (15) with (16), we get

$$
\sum_{i=1}^{15} c_{i \gamma}\left(\left.g_{i}\right|_{\text {out }}-\left.g_{i}\right|_{\text {in }}\right)=\left.\Delta \rho_{\sigma} u_{\gamma}\right|_{\text {in }} \quad \text { for } \quad \gamma=x, y, z,
$$

where $\left.u_{\gamma}\right|_{\text {in }}$ is the $\gamma$-component of the flow velocity at the inlet. Therefore, we finally obtain four equations for four unknowns. The solutions are obtained as follows:

$$
\begin{aligned}
K_{1}= & 3\left[\left.g_{1}\right|_{\text {out }}-\left.g_{1}\right|_{\text {in }}+\left.g_{3}\right|_{\text {out }}-\left.g_{3}\right|_{\text {in }}+\left.g_{4}\right|_{\text {out }}-\left.g_{4}\right|_{\text {in }}\right. \\
& \left.+\left.g_{6}\right|_{\text {out }}-\left.g_{6}\right|_{\text {in }}+\left.g_{7}\right|_{\text {out }}-\left.g_{7}\right|_{\text {in }}-\Delta \rho_{\sigma}\right], \\
K_{2}= & -\left.3 \Delta \rho_{\sigma} u_{x}\right|_{\text {in }}, \\
K_{3}= & 9\left[\left.g_{3}\right|_{\text {out }}-\left.g_{3}\right|_{\text {in }}-\left.g_{6}\right|_{\text {out }}+\left.g_{6}\right|_{\text {in }}-\left.\Delta \rho_{\sigma} u_{y}\right|_{\text {in }}\right], \\
K_{4}= & 9\left[\left.g_{4}\right|_{\text {out }}-\left.g_{4}\right|_{\text {in }}-\left.g_{7}\right|_{\text {out }}+\left.g_{7}\right|_{\text {in }}-\left.\Delta \rho_{\sigma} u_{z}\right|_{\text {in }}\right] .
\end{aligned}
$$


Substituting Equations (27)-(30) into Equations (23) and (24), all the unknown distribution functions for $\sigma$-species at the inlet and outlet are determined for the given $\Delta \rho_{\sigma}$.

In addition, the unknown distribution functions on the corner lines of the inlet and outlet except for the vertices are calculated by the combination of the above-mentioned boundary conditions at the inlet and outlet and those on the sides of the domain. For example, on the lines CF and GJ in Figure 3, taking account of the fact that $u_{z}=0$, we first assume $\left.g_{2}\right|_{\text {in }},\left.g_{11}\right|_{\text {in }},\left.g_{13}\right|_{\text {in }},\left.g_{5}\right|_{\text {out }},\left.g_{12}\right|_{\text {out }}$ and $\left.g_{14}\right|_{\text {out }}$ to be written by the following equations with constant values $K_{5}-K_{7}$ :

$$
\begin{gathered}
\left.g_{i}\right|_{\text {in }}=\left.g_{i}\right|_{\text {out }}+E_{i}\left(K_{5}+c_{i x} K_{6}+c_{i y} K_{7}\right) \quad \text { for } i=2,11,13, \\
\left.g_{i}\right|_{\text {out }}=\left.g_{i}\right|_{\text {in }}-E_{i}\left(K_{5}+c_{i x} K_{6}+c_{i y} K_{7}\right) \quad \text { for } \quad i=5,12,14 .
\end{gathered}
$$

Then, the other unknown distribution functions are expressed by using Equation (18) with parameters $\left.\rho_{\sigma}^{\prime}\right|_{\text {in }}$ and $\left.\rho_{\sigma}^{\prime}\right|_{\text {out }}$. The unknown parameters are determined by Equations (25), (26) for $\gamma=x, y$ and (21) at the inlet and outlet. Hence, we obtain five equations for five unknowns. The solutions are given by

$$
\begin{aligned}
& K_{5}= 3\left[\left.g_{1}\right|_{\text {out }}-\left.g_{1}\right|_{\text {in }}+\left.g_{3}\right|_{\text {out }}-\left.g_{3}\right|_{\text {in }}+\left.g_{6}\right|_{\text {out }}\right. \\
&\left.-\left.g_{6}\right|_{\text {in }}+2\left(\left.g_{7}\right|_{\text {out }}-\left.g_{7}\right|_{\text {in }}\right)-\Delta \rho_{\sigma}\right], \\
& K_{6}=-\left.\frac{18}{5} \Delta \rho_{\sigma} u_{x}\right|_{\text {in }}, \\
& K_{7}= 18\left[\left.g_{3}\right|_{\text {out }}-\left.g_{3}\right|_{\text {in }}-\left.g_{6}\right|_{\text {out }}+\left.g_{6}\right|_{\text {in }}-\left.\Delta \rho_{\sigma} u_{y}\right|_{\text {in }}\right], \\
&\left.\rho_{\sigma}^{\prime}\right|_{\text {in }}= \frac{1}{6}\left(K_{5}+K_{6}\right)+6\left[\left.g_{7}\right|_{\text {in }}+\left.g_{11}\right|_{\text {out }}\right. \\
&\left.\quad+\left.g_{12}\right|_{\text {in }}+\left.g_{13}\right|_{\text {out }}+\left.g_{14}\right|_{\text {in }}\right], \\
&\left.\rho_{\sigma}^{\prime}\right|_{\text {out }}=-\frac{1}{6}\left(K_{5}-K_{6}\right)+6\left[\left.g_{7}\right|_{\text {out }}+\left.g_{11}\right|_{\text {out }}\right. \\
&\left.\quad+\left.g_{12}\right|_{\text {in }}+\left.g_{13}\right|_{\text {out }}+\left.g_{14}\right|_{\text {in }}\right] .
\end{aligned}
$$

Copyright (C) 2000 John Wiley \& Sons, Ltd.

Int. J. Numer. Meth. Fluids 2000; 00:1-6

Prepared using fldauth.cls 
The same method is used on the other corner lines of the inlet and outlet.

\section{RESULTS AND DISCUSSION}

We first consider a mass transfer problem under the condition that $\sigma$-species is diffused from only the body $\mathrm{M}$ whose center is located at $\left(x / L_{x}, y / L_{y}, z / L_{z}\right)=(0.48,0.49,0.49)$ and that the normal mass flux of $\sigma$-species is zero on the other bodies. The computational domain is divided into $73 \times 69 \times 69$ cubic lattice in the $x$-, $y$ - and $z$-directions. The pressure difference $\Delta p$ between the inlet and outlet and the kinematic viscosity $\nu$ of the fluid are changed so that the range of the Reynolds number $R e=\left.\bar{u}\right|_{\text {in }} d_{\mathrm{p}} / \nu$ is $2.21 \leq R e \leq 212$ where $\left.\bar{u}\right|_{\text {in }}$ is the timeand space-averaged velocity at the inlet after transitional flows. Here it should be noted that in the LBM errors caused by the compressibility effect are proportional to the Mach number squared. Hence a small value of $\left.\bar{u}\right|_{\text {in }}$ is desirable, since the errors are proportional to $\left(\left.\bar{u}\right|_{\text {in }}\right)^{2}$. In the following calculations, we choose $\Delta p$ so that $\left.\bar{u}\right|_{\text {in }}$ becomes less than 0.05 in all cases. The Schmidt number $S c=\nu / D_{\sigma}$ is fixed at 1 . The concentration of $\sigma$-species on the body M is kept at $\left.\rho_{\sigma}\right|_{\mathrm{w}}=2.5$. The concentration difference of $\sigma$-species between the inlet and outlet is fixed at $\Delta \rho_{\sigma}=0.1$. The initial conditions for the macroscopic variables are $\rho=1, \mathbf{u}=\mathbf{0}$, $\rho_{\sigma}=1$ and $\mathbf{j}_{\sigma}=\mathbf{0}$ in the whole domain.

As in [15], when preliminary computations with the coarser grids $(38 \times 35 \times 35$ and $49 \times 46 \times 46$ cubic lattices) were performed, numerical instabilities occurred and the computations did not converge at high Reynolds numbers, though almost grid-independent results were obtained at lower Reynolds numbers. Also, the computation time for the present calculation with $73 \times 69 \times 69$ cubic lattices required about 100 minutes per 1,000 time steps on a single processor of the COMPAQ Alpha Station with $667 \mathrm{MHz}$ clock speed.

Copyright (C) 2000 John Wiley \& Sons, Ltd.

Int. J. Numer. Meth. Fluids 2000; 00:1-6 
Figures 4-6 show the calculated results of flow velocity vectors (left) and concentration profiles of $\sigma$-species (right) on the different planes $\left(y / L_{y}=0.36, y / L_{y}=0.88\right.$ and $\left.x / L_{x}=0.51\right)$ at various Reynolds numbers ( $R e=2.21,38.1$ and 212$)$ after transitional flows. In these figures, the length of vectors is normalized so that the $\left.\bar{u}\right|_{\text {in }}$ has the same length in spite of different Reynolds numbers, and the bodies in the structure are depicted by the spheres with the equivalent diameter $d_{\mathrm{p}}=29.4 \Delta x$. Also, it is noted that in Figures 4 and 6 the dark gray body in the center represents the body M from which $\sigma$-species is diffused. Figures 4 and 5 show the results on the two different planes parallel to main flow. It is found from Figure 4 that at low Reynolds number of $R e=2.21$ the fluid flow avoids the bodies and goes through open spaces, and the $\sigma$-species spreads in every direction mainly by the effect of diffusion. At moderate Reynolds number of $R e=38.1$, on the other hand, it is seen that the flow speed becomes a little larger and $\sigma$-species is diffused together with fluid flow. At high Reynolds number of $R e=212$ the flow separations occur and several vortices appear behind the bodies, and the concentration of $\sigma$-species is highly affected by the flow convection. In addition, $\sigma$-species diffuses mainly through the central region of the domain. Figure 5 shows the calculated results on the different plane. It should be noted that this plane has no cross-section of the body M. At $R e=2.21$ the concentration of $\sigma$-species increases almost linearly in the $x$-direction. On the contrary, at $R e=212$ the concentration of $\sigma$-species varies three-dimensionally and is completely different from those at lower Reynolds numbers. Figure 6 shows the results on the plane vertical to main flow. It is seen that at $R e=2.21$ the concentration of $\sigma$-species is almost uniform on the $y-z$ plane, while at $R e=212$ the steep concentration gradient exists around the body $\mathrm{M}$ and the concentration profile becomes more complicated.

We next consider a mass transfer problem in Figure 3 under the condition that $\sigma$-species is Copyright (C) 2000 John Wiley \& Sons, Ltd. Int. J. Numer. Meth. Fluids 2000; 00:1-6 Prepared using fldauth.cls 
diffused from all the bodies in the structure. In the following, , the concentration of $\sigma$-species at the boundary nodes on the bodies is increased linearly from the inlet to outlet. The value of $\left.\rho_{\sigma}\right|_{\mathrm{w}}$ at the inlet is kept at 1 . The concentration difference $\Delta \rho_{\sigma}$ between the inlet and outlet is fixed at 0.5. In addition, the Schmidt number is fixed at 0.6. The other conditions are the same as the previous ones. The Sherwood number Sh between the inlet and outlet can be calculated by the following equation:

$$
S h=\frac{\Delta \rho_{\sigma}}{\left(\left.\rho_{\sigma}\right|_{\mathrm{w}}-\bar{\rho}_{\sigma \mathrm{m}}\right)_{\mathrm{in}}} \operatorname{Re} S c \frac{L_{y} L_{z}}{S_{\mathrm{tot}}}
$$

where $\bar{\rho}_{\sigma \mathrm{m}}$ is the bulk concentration of $\sigma$-species, and $S_{\text {tot }}$ is the total surface area of all the bodies in the structure. Figure 7 shows the comparison of calculated results with experimental data $(S c=0.6)$ for packed beds by Petrovic and Thodos [32]. In Figure 7, the solid circle and the cross indicate the calculated results and the experimental data, respectively. It is found from this figure that the calculated values agree well with the experimental data at low and high Reynolds numbers.

\section{CONCLUDING REMARKS}

The LBM for a binary miscible fluid mixture is applied to the problems of transport phenomena in a three-dimensional porous structure. Flow characteristics and concentration profiles of diffusing species are obtained at various Reynolds numbers. The Sherwood numbers are calculated and compared with the experimental data for packed beds. The results indicate that the calculated Sherwood numbers are in good agreement with experimental data at low and high Reynolds numbers. Finally, it should be noted that by introducing other particle distribution functions, one can perform the calculations for the problems of simultaneous 
heat/mass transfer and of multicomponent convection-diffusion systems in porous structures. Therefore, the present method is useful for the investigation of transport phenomena in porous structures.

\section{ACKNOWLEDGEMENTS}

The authors would like to thank Professor F. Ogino and Professor Y. Matsuda for their valuable discussions on this research. Cordial thanks are due to the anonymous referees for their helpful comments. The authors also thank Y. Takeuchi and K. Ichino for assistance in some computations and in drawing figures.

\section{REFERENCES}

1. Ergun S. Fluid flow through packed columns. Chem. Eng. Prog. 1952; 48: 89-94.

2. Vafai K, Tien CL. Boundary and inertia effects on flow and heat transfer in porous media. Int. J. Heat Mass Transfer 1981; 24: 195-203.

3. Buonanno G, Carotenuto A. The effective thermal conductivity of a porous medium with interconnected particles. Int. J. Heat Mass Transfer 1997; 40: 393-405.

4. Koch DL, Brady JF. Dispersion in fixed beds. J. Fluid Mech. 1985; 154: 399-427.

5. Koch DL, Cox RG, Brenner H, Brady JF. The effect of order on dispersion in porous media. J. Fluid Mech. 1989; 200: 173-188.

6. Hsu CT, Cheng P. Thermal dispersion in a porous medium. Int. J. Heat Mass Transfer 1990; 33: 15871597.

7. Salles J, Thovert J.-F, Delannay R, Prevors L, Auriault J.-L, Adler PM. Taylor dispersion in porous media. Determination of the dispersion tensor. Phys. Fluids A 1993; 5: 2348-2376.

8. McNamara GR, Zanetti G. Use of the Boltzmann equation to simulate lattice-gas automata. Phys. Rev. Lett. 1988; 61: 2332-2335.

9. Higuera FJ, Jimenez J. Boltzmann approach to lattice gas simulations, Europhys. Lett. 1989; 9: 663-668.

10. Chen S, Chen H, Martínez D, Matthaeus WH. Lattice Boltzmann model for simulation of magnetohydrodynamics, Phys. Rev. Lett. 1991; 67: 3776-3779.

Copyright (C) 2000 John Wiley \& Sons, Ltd.

Int. J. Numer. Meth. Fluids 2000; 00:1-6

Prepared using fldauth.cls 
11. Qian YH, d'Humières D, Lallemand P. Lattice BGK models for Navier-Stokes equation. Europhys. Lett. 1992; 17: 479-484.

12. Chen S, Doolen GD. Lattice Boltzmann method for fluid flows. Annu. Rev. Fluid Mech. 1998; 30: 329-364.

13. Succi S. The lattice Boltzmann equation for fluid dynamics and beyond. (Oxford University Press, Oxford, UK, 2001).

14. Cancelliere A, Chang C, Foti E, Rothman DH, Succi S. The permeability of a random medium: comparison of simulation with theory. Phys. Fluids A 1990; 2: 2085-2088.

15. Inamuro T, Yoshino M, Ogino F. Lattice Boltzmann simulation of flows in a three-dimensional porous structure. Int. J. Numer. Meth. Fluids 1999; 29: 737-748.

16. Inamuro T, Yoshino M, Ogino F. Numerical analysis of unsteady flows in a three-dimensional porous structure. Kagaku Kogaku Ronbunshu 1999; 25: 979-986, (in Japanese).

17. Zeiser T, Steven M, Freund H, Lammers P, Brenner G, Durst F, Bernsdorf J. Analysis of the flow field and pressure drop in fixed-bed reactors with the help of lattice Boltzmann simulations. Phil. Trans. R. Soc. Lond. A 2002; 360: 507-520.

18. Flekkøy EG. Lattice Bhatnagar-Gross-Krook models for miscible fluids. Phys. Rev. E 1993; 47: 4247-4257.

19. Shan X, Doolen G. Diffusion in a multicomponent lattice Boltzmann equation model. Phys. Rev. E 1996; 54: $3614-3620$.

20. McNamara GR, Garcia AL, Alder BJ. A hydrodynamically correct thermal lattice Boltzmann model. $J$. Stat. Phys. 1997; 87: 1111-1121.

21. Shan X. Simulation of Rayleigh-Bénard convection using a lattice Boltzmann method. Phys. Rev. E 1997; 55: $2780-2788$.

22. He X, Chen S, Doolen GD. A novel thermal model for the lattice Boltzmann method. J. Comput. Phys. 1998; 146: 282-300.

23. Inamuro T, Yoshino M, Inoue H, Mizuno R, Ogino F. A lattice Boltzmann method for a binary miscible fluid mixture and its application to a heat-transfer problem. J. Comput. Phys. 2002; 179: 201-215.

24. Ferziger JH, Perić M. Computational methods for fluid dynamics. (Springer-Verlag, Berlin, Germany, 1996).

25. Inamuro T, Yoshino M, Ogino F. Accuracy of the lattice Boltzmann method for small Knudsen number with finite Reynolds number. Phys. Fluids 1997; 9: 3535-3542.

26. Sone Y. Asymptotic theory of flow of rarefied gas over a smooth boundary II. In Rarefied Gas Dynamics, Dini D, (ed). vol. 2. Editrice Tecnico Scientifica, Pisa, 1971; 737-749.

Copyright (C) 2000 John Wiley \& Sons, Ltd.

Int. J. Numer. Meth. Fluids 2000; 00:1-6

Prepared using fldauth.cls 
27. Inamuro T. A lattice kinetic scheme for incompressible viscous flows with heat transfer. Phil. Trans. R. Soc. Lond. A 2002; 360: 477-484.

28. Nadiga BT. A study of multi-speed discrete-velocity gases. Ph.D. thesis (California Institute of Technology, 1992).

29. Hortmann M, Perić M, Scheuerer G. Finite volume multigrid prediction of laminar natural convection: bench-mark solutions. Int. J. Numer. Meth. Fluids 1990; 11: 189-207.

30. Patankar SV. Numerical heat transfer and fluid flow. (McGraw-Hill, New York, 1980).

31. Inamuro T, Yoshino M, Ogino F. A non-slip boundary condition for lattice Boltzmann simulations. Phys. Fluids 1995; 7: 2928-2930; Erratum 8: 1124.

32. Petrovic LJ, Thodos G. Mass transfer in the flow of gases through packed beds - low Reynolds number region. Ind. Eng. Chem. Fund. 1968; 7: 274-280. 


\section{FIGURES}

Figure 1. Geometry of natural convection example problem.

Figure 2. Calculated results for $R a=10^{5}$ and $P r=0.71$ on an $80 \times 80$ lattice: (a) velocity vectors; (b) isotherms.

Figure 3. Three-dimensional porous structure.

Figure 4. Flow velocity vectors (left) and concentration profiles of $\sigma$-species (right) on the plane of $y / L_{y}=0.36$ at various Reynolds numbers: (a) $R e=2.21, \Delta \Pi=4.25 \times 10^{-1}, \delta \rho_{\sigma}=1.18 \times 10^{-2}$; (b) $R e=38.1, \Delta \Pi=6.55 \times 10^{-2}, \delta \rho_{\sigma}=7.63 \times 10^{-2} ; \quad(\mathrm{c}) R e=212, \Delta \Pi=4.56 \times 10^{-2}, \delta \rho_{\sigma}=1.10 \times 10^{-1}$, where $\Delta \Pi=\Delta \rho_{\sigma} /\left(\left.\rho_{\sigma}\right|_{\mathrm{w}}-\left.\rho_{\sigma \mathrm{m}}\right|_{\text {in }}\right)$, and $\delta \rho_{\sigma}$ is a contour interval. The dark gray body in the center is M from which $\sigma$-species is diffused.

Figure 5. Flow velocity vectors (left) and concentration profiles of $\sigma$-species (right) on the plane of $y / L_{y}=0.88$ at various Reynolds numbers: (a) $R e=2.21, \Delta \Pi=4.25 \times 10^{-1}, \delta \rho_{\sigma}=1.18 \times 10^{-2}$; (b) $R e=38.1, \Delta \Pi=6.55 \times 10^{-2}, \delta \rho_{\sigma}=7.63 \times 10^{-2} ; \quad(\mathrm{c}) R e=212, \Delta \Pi=4.56 \times 10^{-2}, \delta \rho_{\sigma}=1.10 \times 10^{-1}$, where $\Delta \Pi=\Delta \rho_{\sigma} /\left(\left.\rho_{\sigma}\right|_{\mathrm{w}}-\left.\rho_{\sigma \mathrm{m}}\right|_{\text {in }}\right)$, and $\delta \rho_{\sigma}$ is a contour interval. This plane has no cross-section of the body $\mathrm{M}$.

Figure 6. Flow velocity vectors (left) and concentration profiles of $\sigma$-species (right) on the plane of $x / L_{x}=0.51$ at various Reynolds numbers: (a) $R e=2.21, \Delta \Pi=4.25 \times 10^{-1}, \delta \rho_{\sigma}=1.18 \times 10^{-2}$; (b) $R e=38.1, \Delta \Pi=6.55 \times 10^{-2}, \delta \rho_{\sigma}=7.63 \times 10^{-2} ; \quad(\mathrm{c}) R e=212, \Delta \Pi=4.56 \times 10^{-2}, \delta \rho_{\sigma}=1.10 \times 10^{-1}$, where $\Delta \Pi=\Delta \rho_{\sigma} /\left(\left.\rho_{\sigma}\right|_{\mathrm{w}}-\left.\rho_{\sigma \mathrm{m}}\right|_{\text {in }}\right)$, and $\delta \rho_{\sigma}$ is a contour interval. The dark gray body in the center is $\mathrm{M}$ from which $\sigma$-species is diffused.

Figure 7. Comparison of calculated Sherwood numbers with experimental data: $\bullet$, the present calculated results; $\times$, the experimental data by Petrovic and Thodos [32].

Copyright (c) 2000 John Wiley \& Sons, Ltd.

Int. J. Numer. Meth. Fluids 2000; 00:1-6

Prepared using fldauth.cls 


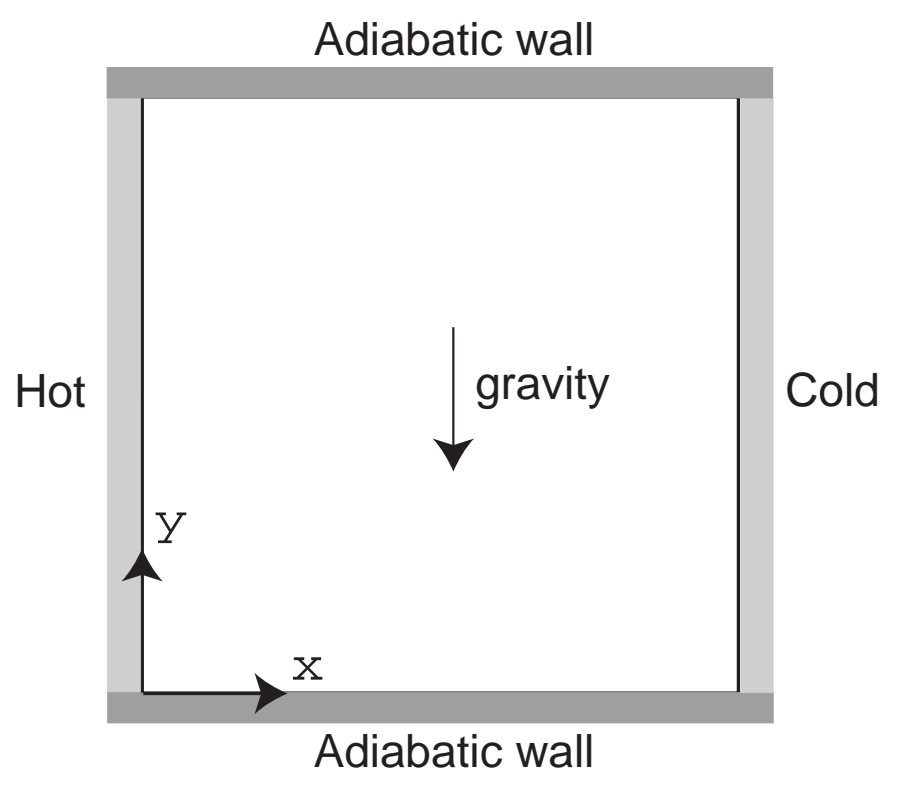

Figure 1. Geometry of natural convection example problem. 


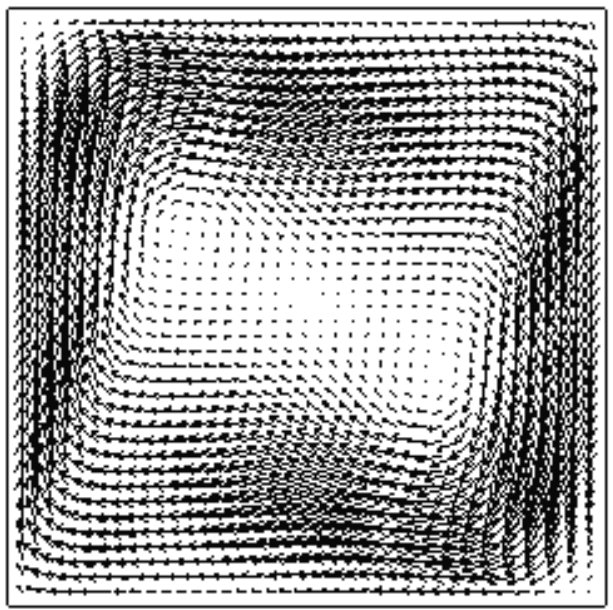

(a)

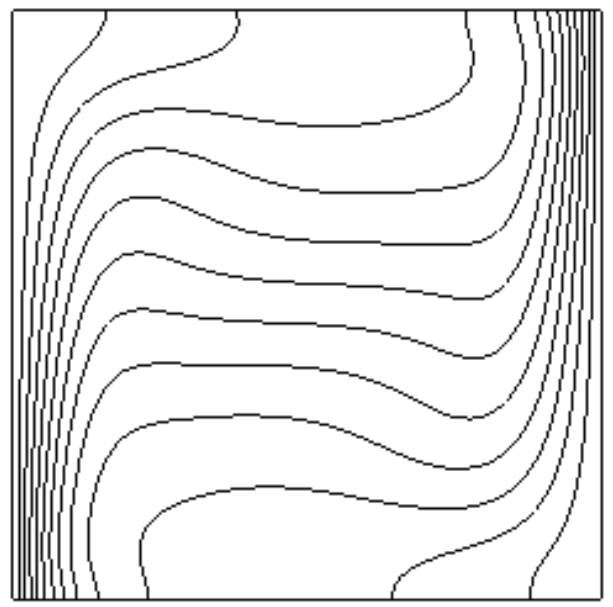

(b)

Figure 2. Calculated results for $R a=10^{5}$ and $\operatorname{Pr}=0.71$ on an $80 \times 80$ lattice: (a) velocity vectors; (b) isotherms. 


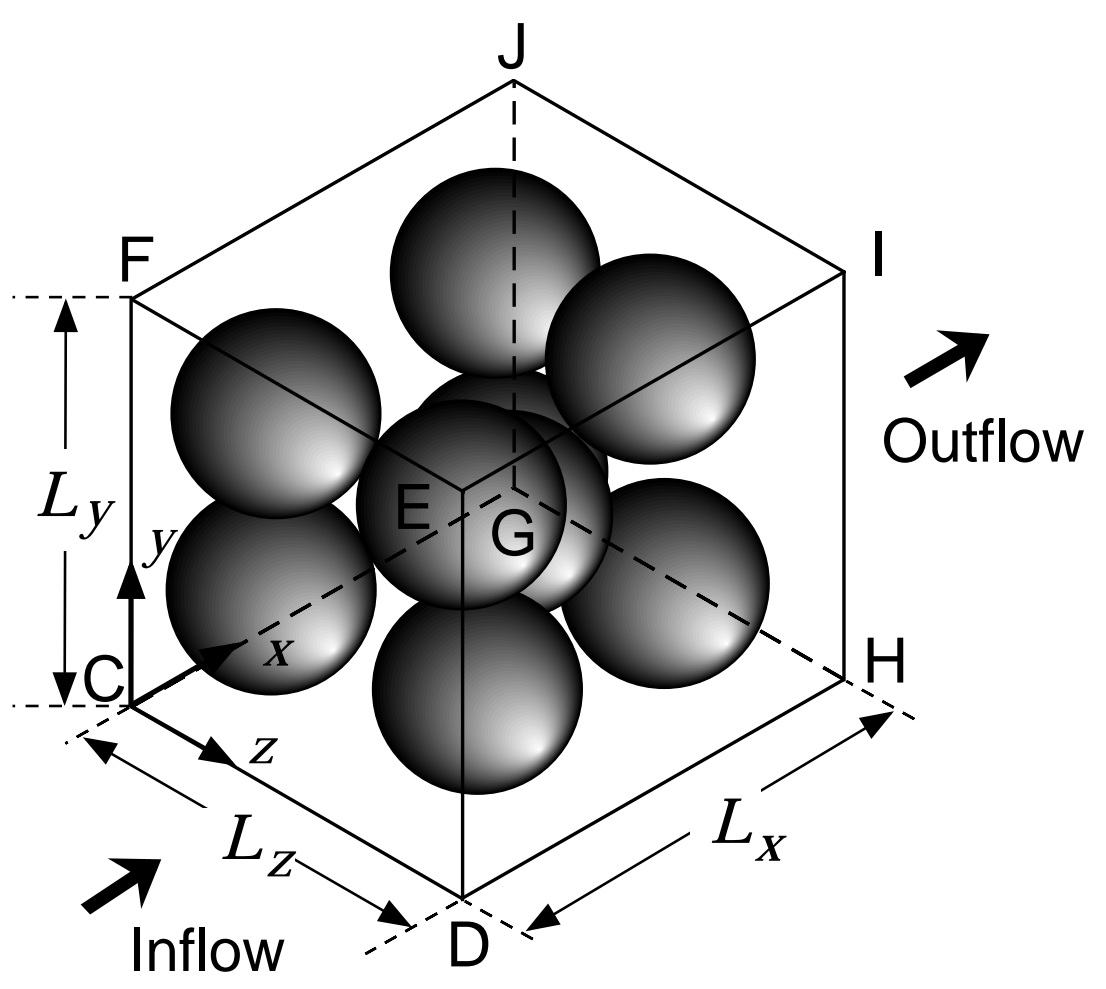

Figure 3. Three-dimensional porous structure. 
$\left.\longrightarrow 5 \overline{\mathrm{u}}\right|_{\text {in }}$

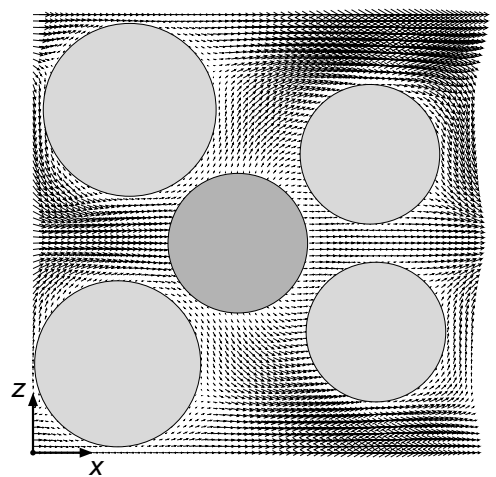

$\left.\rightarrow 5 \overline{\mathrm{u}}\right|_{\text {in }}$

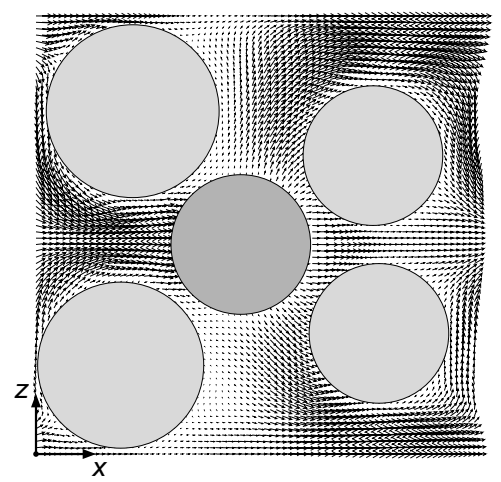

$\left.\rightarrow 5 \overline{\mathrm{u}}\right|_{\text {in }}$

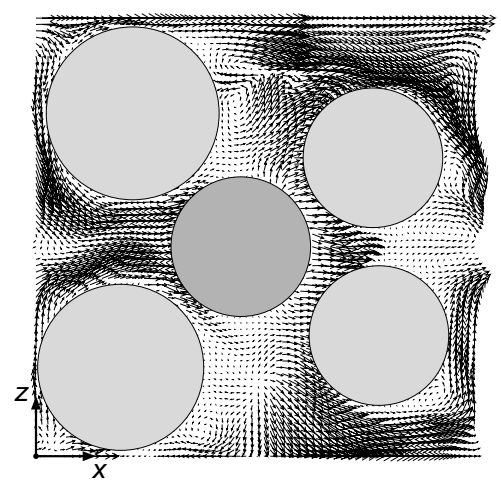

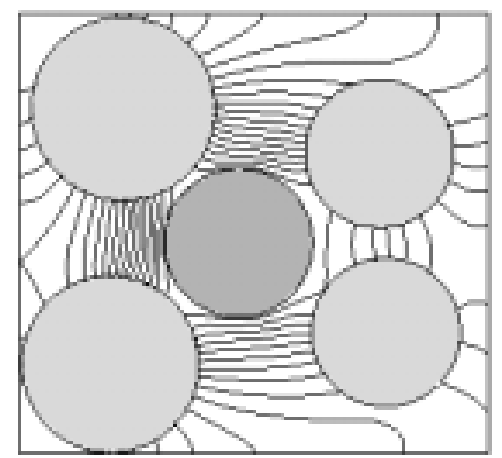

(a)

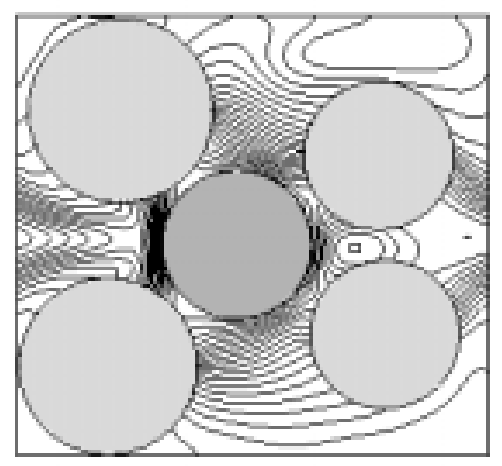

(b)

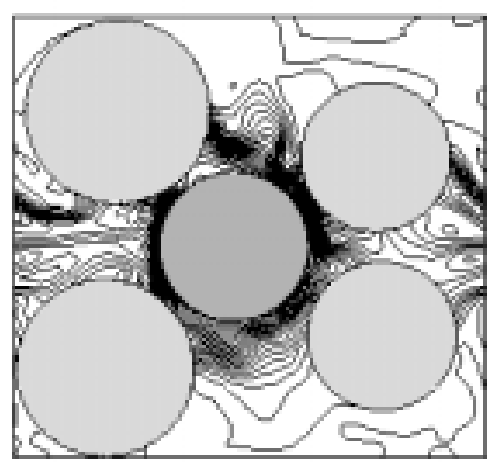

(c)

Figure 4. Flow velocity vectors (left) and concentration profiles of $\sigma$-species (right) on the plane of $y / L_{y}=0.36$ at various Reynolds numbers: (a) $R e=2.21, \Delta \Pi=4.25 \times 10^{-1}, \delta \rho_{\sigma}=1.18 \times 10^{-2}$; (b) $R e=38.1, \Delta \Pi=6.55 \times 10^{-2}, \delta \rho_{\sigma}=7.63 \times 10^{-2} ; \quad(\mathrm{c}) R e=212, \Delta \Pi=4.56 \times 10^{-2}, \delta \rho_{\sigma}=1.10 \times 10^{-1}$, where $\Delta \Pi=\Delta \rho_{\sigma} /\left(\left.\rho_{\sigma}\right|_{\mathrm{w}}-\left.\rho_{\sigma \mathrm{m}}\right|_{\text {in }}\right)$, and $\delta \rho_{\sigma}$ is a contour interval. The dark gray body in the center is M from which $\sigma$-species is diffused.

Copyright (C) 2000 John Wiley \& Sons, Ltd.

Int. J. Numer. Meth. Fluids 2000; 00:1-6 Prepared using fldauth.cls 
$>\left.5 \bar{u}\right|_{\text {in }}$

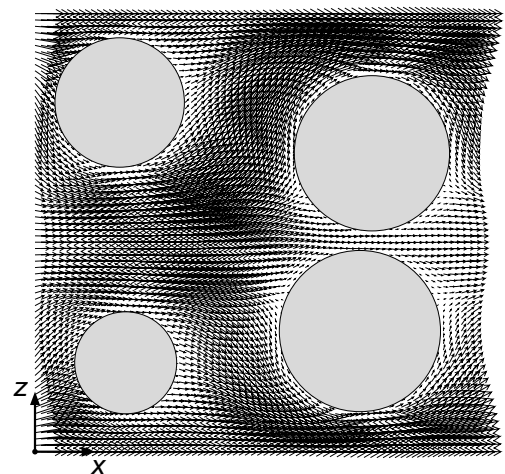

$\left.\rightarrow 5 \bar{u}\right|_{\text {in }}$

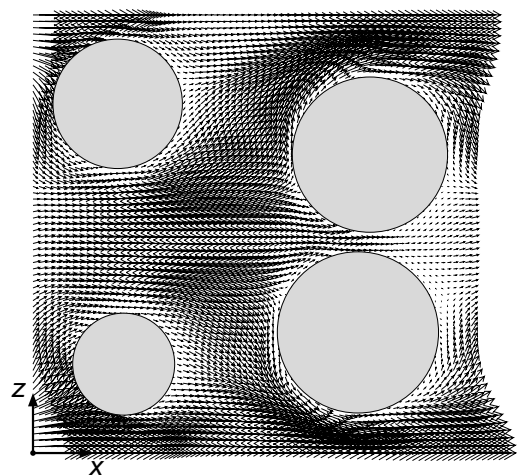

$\left.\rightarrow 5 \bar{u}\right|_{\text {in }}$

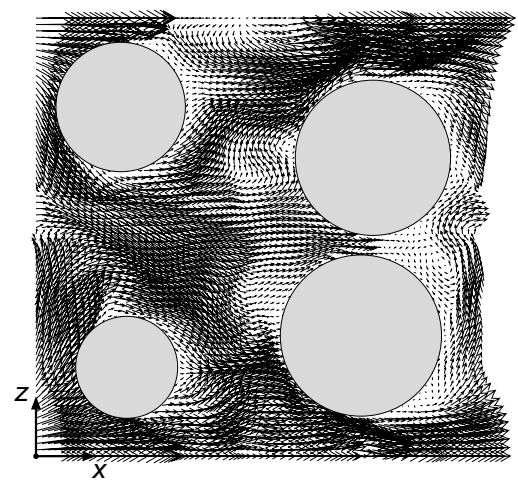

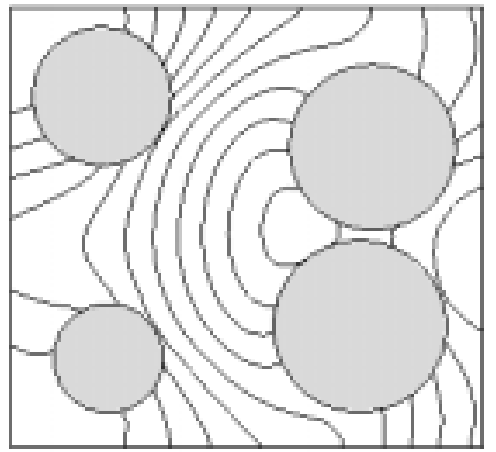

(a)

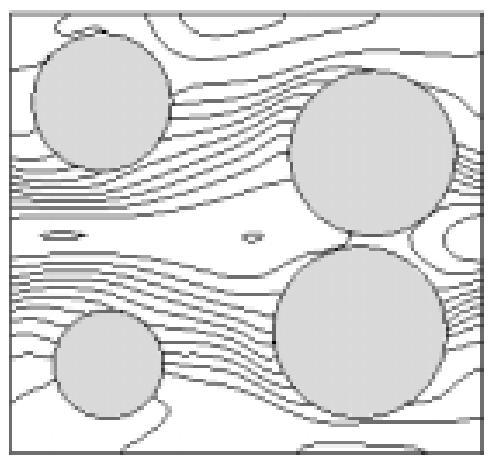

(b)

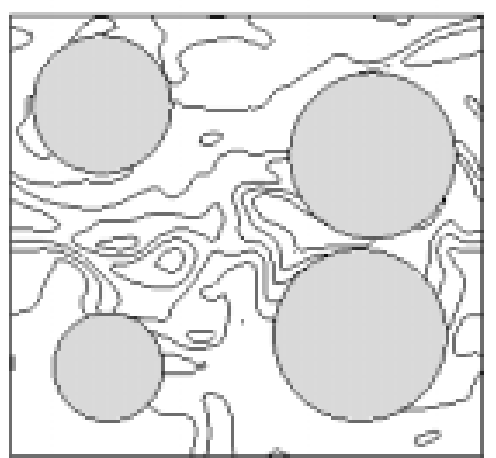

(c)

Figure 5. Flow velocity vectors (left) and concentration profiles of $\sigma$-species (right) on the plane of $y / L_{y}=0.88$ at various Reynolds numbers: (a) $R e=2.21, \Delta \Pi=4.25 \times 10^{-1}, \delta \rho_{\sigma}=1.18 \times 10^{-2} ;$ (b) $R e=38.1, \Delta \Pi=6.55 \times 10^{-2}, \delta \rho_{\sigma}=7.63 \times 10^{-2} ; \quad\left(\right.$ c) $R e=212, \Delta \Pi=4.56 \times 10^{-2}, \delta \rho_{\sigma}=1.10 \times 10^{-1}$, where $\Delta \Pi=\Delta \rho_{\sigma} /\left(\left.\rho_{\sigma}\right|_{\mathrm{w}}-\left.\rho_{\sigma \mathrm{m}}\right|_{\text {in }}\right)$, and $\delta \rho_{\sigma}$ is a contour interval. This plane has no cross-section of the body $\mathrm{M}$.

Copyright (C) 2000 John Wiley \& Sons, Ltd.

Int. J. Numer. Meth. Fluids 2000; 00:1-6

Prepared using fldauth.cls 
$\left.\longrightarrow 5 \bar{u}\right|_{\text {in }}$

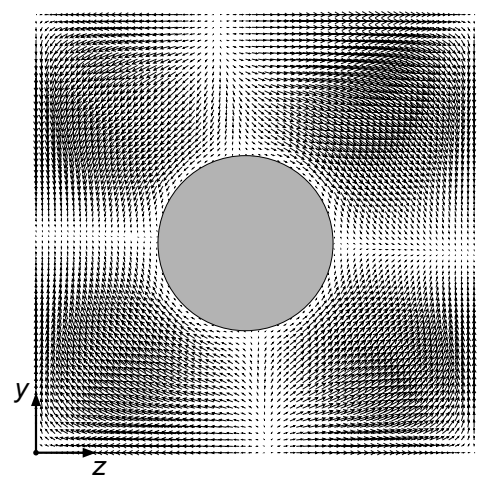

$\left.\longrightarrow 5 \bar{u}\right|_{\text {in }}$

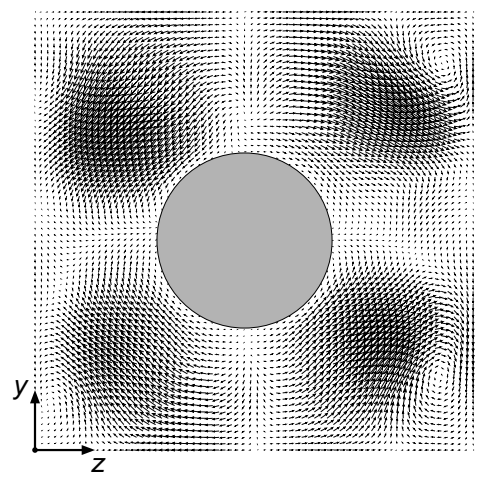

$\left.\longrightarrow 5 \bar{u}\right|_{\text {in }}$

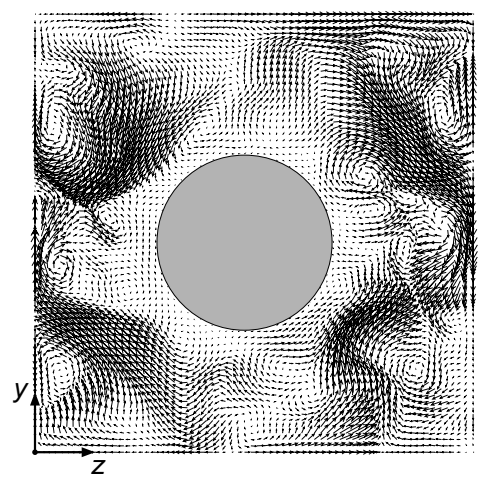

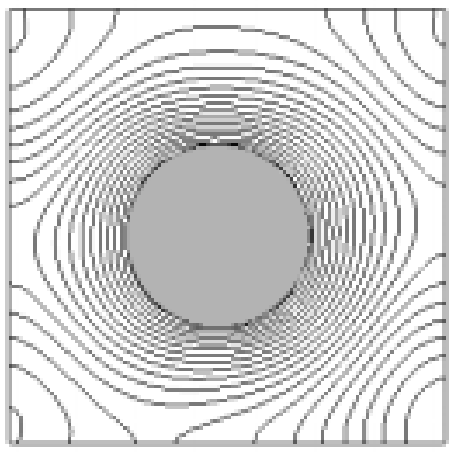

(a)

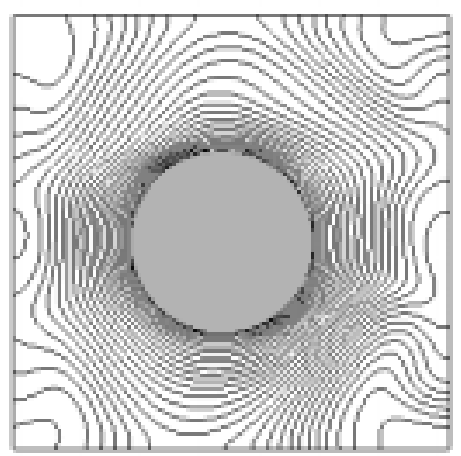

(b)

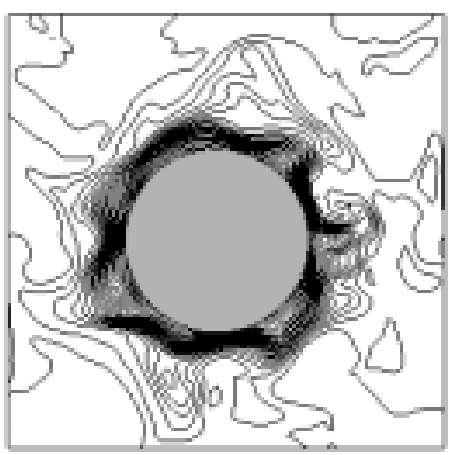

(c)

Figure 6. Flow velocity vectors (left) and concentration profiles of $\sigma$-species (right) on the plane of $x / L_{x}=0.51$ at various Reynolds numbers: (a) $R e=2.21, \Delta \Pi=4.25 \times 10^{-1}, \delta \rho_{\sigma}=1.18 \times 10^{-2}$; (b) $R e=38.1, \Delta \Pi=6.55 \times 10^{-2}, \delta \rho_{\sigma}=7.63 \times 10^{-2} ; \quad\left(\right.$ c) $R e=212, \Delta \Pi=4.56 \times 10^{-2}, \delta \rho_{\sigma}=1.10 \times 10^{-1}$, where $\Delta \Pi=\Delta \rho_{\sigma} /\left(\left.\rho_{\sigma}\right|_{\mathrm{w}}-\left.\rho_{\sigma \mathrm{m}}\right|_{\text {in }}\right)$, and $\delta \rho_{\sigma}$ is a contour interval. The dark gray body in the center is $\mathrm{M}$ from which $\sigma$-species is diffused.

Copyright (C) 2000 John Wiley \& Sons, Ltd.

Int. J. Numer. Meth. Fluids 2000; 00:1-6

Prepared using fldauth.cls 


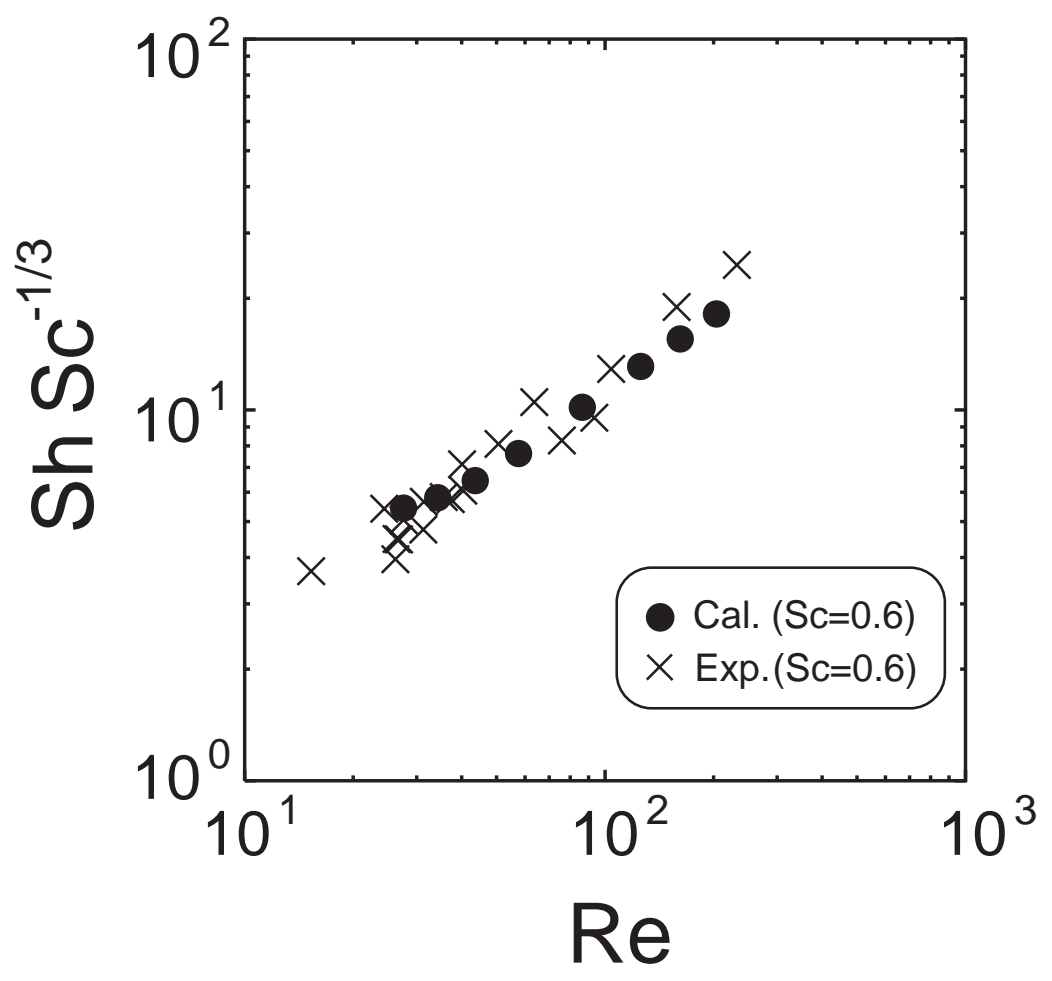

Figure 7. Comparison of calculated Sherwood numbers with experimental data: $\bullet$, the present calculated results; $\times$, the experimental data by Petrovic and Thodos [32]. 